\title{
NOSOCOMIAL INFECTIONS AND THE CHALLENGES OF CONTROL IN
} DEVELOPING COUNTRIES.

\author{
Samuel,S. O ${ }^{1}$, Kayode,O. O ${ }^{2}$, Musa,O. I ${ }^{2}$, Nwigwe,G.C ${ }^{3}$, Aboderin A.O, ${ }^{4}$ \\ Salami T.A.T, ${ }^{5}$ Taiwo S.S ${ }^{6}$
}

Departments of ${ }^{1}$ Medical Microbiology and Parasitology, ${ }^{5}$ Medicine, Faculty of Clinical Sciences, College of Medicine, Ambrose Alli University, Ekpoma, Nigeria.

${ }^{2}$ Department of Epidemiology and Community Health, Faculty of Clinical Sciences, College of Health Sciences, University Ilorin, Nigeria.

${ }^{3}$ Department of Surgery, Faculty of Clinical Medicine, College of Health Sciences, Ebonyi State University, Abakaliki, Nigeria.

${ }^{4}$ Department of Medical Microbiology and Parasitology, Obafemi Awolowo University Ile - Ife, Nigeria

${ }^{6}$ Department of Medical, Microbiology and Parasitology, College of Health Sciences, Ladoke Akintola University College of Technology Teaching Hospital, Oshogbo, Nigeria.

Correspondence : Dr. S. O. Samuel Department of Medical Microbiology and Parasitology, Faculty of Clinical Sciences College of Medicine, Ambrose Alli University, Ekpoma, Nigeria. E-mail: olowosamuel2003@yahoo.com

\begin{abstract} health workers. risks of cross infection.

\section{INTRODUCTION}

Nosocomial infections, otherwise known as hospital-acquired infections are those infections acquired in hospital or healthcare service unit, that first appear 48 hours or more after hospital admission or within 30 days after discharge following in patient care (1). They are unrelated to the original illness that brings patients to the hospital and neither present nor incubating as at the time of admission. $(1,2)$. There are several reasons why nosocomial infections are even more alarming in the $21^{\text {st }}$ century. These include hospitals housing large number of people who are sick and whose immune system are often in a weakened state, increased use of
\end{abstract}

Nosocomial infection is a recognized public health problem world-wide with a prevalence rate of $3.0-20.7 \%$ and an incidence rate of 5-10\%. It has become increasingly obvious that infections acquired in the hospital lead to increased morbidity and mortality which has added noticeably to economic burden.

However, after about three decades of nosocomial infection surveillance and control world-wide, it still remains an important problem for hospitals today. Studies have shown that most hospitals in developing countries especially Africa, have no effective infection control programme due to lack of awareness of the problem, lack of personnel, poor water supply, erratic electricity supply, ineffective antibiotic policies with emergence of multiply antibiotic resistant microbes, poor laboratory backup, poor funding and non-adherence to safe practices by

It is recommended that the cost of hospital infection control programme should be included in the health budget of the country and fund allocated for the infection control committee for routine control purposes and to bear the cost of outbreaks. There is need for adequate staffing and continuous education of staff on the principles of infection control, especially hand washing which is the single most important effective measure to reduce the outpatient treatment meaning that people who are in hospital are sicker on average, many medical procedures that bypass the body's natural protective barriers, medical staff move from patient to patient thus providing a way for

pathogens to spread, inadequate sanitation protocols regarding uniforms ,equipment sterilization, washing and other preventive measures that may either be unheeded by hospital personnel or too lax to sufficiently isolate patients from infectious agents and lastly the routine use of anti-microbial agents in hospitals creates selection pressure for the emergence of the resistant strains of microorganisms(1). Nosocomial infections may range from mild to severe with an incidence of $5-10 \%(2)$. A WHO prevalence study puts its 
prevalence rate at $3.0-20.7 \% \%^{3}$ Hospital infection control programs can prevent $33 \%$ of nosocomial infections (2).

The significance of nosocomial infection lies not only in its ability to substantially alter morbidity and mortality statistics, but also in its economic implications. Nosocomial infection prolongs duration of hospitalization, increases the cost of health care, emergence of multiple antibiotic resistance microorganisms and reduces the chances of treatment for others $(4,5,6)$. Nosocomial infections pose a problem of enormous magnitude globally, hospital localities have proven favourable in transmitting infections due to existing suitable pathogen-hostenvironment relationship. Estimates from various countries show that at any point in time, a significant number of hospitalized patients develop infections which were not present or incubating when the patients were admitted to hospitals. Furthermore, it has become increasingly obvious that such infections acquired in hospital add noticeably to morbidity and economic burden (7). Realizing the importance of this to world health, various international organizations including accreditation and governmental agencies, national associations and organizations, World Health Organization, United Nations Environmental programme, United Nations Children's Fund and various countries have made commendable efforts in checking these infections. ${ }^{7}$ However, after about three decades of nosocomial infections surveillance and control in hospitalized patients world-wide, nosocomial infections remain an important problem for hospitals today (7). This review is important considering the increase in morbidity and mortality due to hospital acquired infections, coupled with the lack of effective infection control programes in some hospitals especially in developing countries due to poor adherence to safe practices by health workers and also lack of personnel trained in infection control practices. There is need for renewed efforts geared towards education through training and re-training coupled with research to keep nosocomial infections in check (7). The objective of this review is to present an overview of nosocomial infections, identify the major challenges of control in developing countries and make appropriate recommendations aimed at effective control.

\section{HISTORICAL PERSPECTIVE}

Nosocomial infections must have existed from the time a number of people with various ailments were brought together for care, but were only readily acknowledged in the middle of the $19^{\text {th }}$ century. During this period, some clinicians notably Oliver Wendel Holmes in Boston and Ignaz Philip Semmelweis in Vienna emphasized on the contagious nature of puerperal sepsis or child - bed fever $(8,9)$. Semmelweis noticed that about $8.3 \%$ of women admitted to maternity services died of nosocomial puerperal sepsis. He was able to demonstrate a dramatic reduction to $2.3 \%$ in the incidence of this disease by insisting on stringent hand washing in a solution of chlorinated lime by students coming from the postmortem room before the women could be examined. Joseph Lister recognized the significance of bacteria in producing postoperative wound infections, and successfully attempted the use of carbolic acid in treating compound fractures and wounds $(10,11)$. These observations and innovations provided the basis for the epidemiology and control of nosocomial infections and these authors were the forerunners of modern aseptic techniques. The introduction of antimicrobial agents specifically penicillin into chemotherapeutics in 1941 brought a wave of premature optimism that, diseases, especially those acquired in hospital could be readily treated (11). Unfortunately, interest in infection control practices rapidly waned. In the 1940s and 1950s, severe world-wide Staphylococcus aureus pandemics caused substantial morbidity and mortality in hospitals, resulting in a rebirth of the need to establish infection control programmes in hospitals $(11,12)$. The joint commission on Accreditation of Health Care Organization (JCAHO) in the United States of America, in part due to the pandemics, recommended the appointment of infection control committees by hospitals (13).

The field of hospital infection was first conceptualized and implemented in England in late 1950s (1959) when Dr. Brendan Moore, Director of the Public Health Laboratory in Exeter, had a nurse in today hospital appointed as an "infection control sister" 14). In 1960, Dr. Moore still initiated the appointment of a second sister at the Exeter hospital. The mission of the two nurses was to improve efforts in combating and controlling the widespread problem of nosocomial infection. In 1963, the United States followed England's lead into the field of infection control and appointed the first control 
nurse, Kathryn Wenzel, a registered nurse at Stanford university, California 11). The appointment of infection control nurses in the United Kingdom and the United States marked the beginning of a new era, the recognition of infection control as a specialty in its own right. The discipline of hospital infection control was thus born out of sheer determination to minimize infections in hospitalized patients and to help optimize patient care. During the 1960s, control procedures in many hospitals were random and non specific in approach. They consisted mainly of routine microbiologic culturing of air and environmental surfaces such as floors, walls and table tops in various areas of the Hospital. In the 1970s, such practices were critically evaluated and emphasis shifted away from the environment to the surveillance of infections in patients (17).

The hospital-wide surveillance method, also known as comprehensive or total surveillance, was used whereby all patients were monitored for nosocomial infections at body sites, and overall rates of infection were calculated (18). This system remained essentially unchanged until 1986 when the need for more precise measurements of nosocomial infection risks and outcome in specific patients groups led to the introduction of three other surveillance methods, viz adult and paediatric intensive care unit, high risk nursery and surgical patients method. The four techniques may be used singly or simultaneously and are designed to form the foundation of a hospital's overall surveillance programme. They also allow flexibility for incorporating other aspects into the surveillance efforts. As part of this revision, definitions of nosocomial infections were expanded to include clinical and laboratory algorithms (19).

\section{EPIDEMIOLOGY}

All over the world, nosocomial infection is a recognized public health problem, Surveillance programmes estimate the rate of infection at 5-10 $\%$ of hospital admissions (1, 20, 21, 22, 23). Nosocomial infections are responsible for about 90,000 deaths in the U.S. per year and approximately $10 \%$ of American hospital patients (about 2 million every year) acquired a clinically significant nosocomial infections ${ }^{23}$. Estimates of the annual cost range from \$4.5-11 billion $^{4,24}$. In France, the prevalence is of nosocomial infections $6.9 \%$ to $7.5 \%$. A rate of 5 to $19 \%$ hospitalized patients are infected and up to $30 \%$ are in intensive care units $(25,26)$. In
Italy in 2000s, about $6.7 \%$ of hospitalized patients were infected; that means, between 450,000 and 700,000 patients had nosocomial infections out of which between 4,500 and 7000 died $(26,27)$. In Switzerland, extrapolations assume about 70,000 hospitalized patients affected by nosocomial infections (between 2 and $14 \%$ of hospitalized patients (27). In Nigeria, nosocomial infection rate of $2.7 \%$ was reported from Ife (28), while $3.8 \% 29$ from Lagos and $4.2 \%$ from Ilorin 30 ) The cause of nosocomial infections might be endogenous or exogenous. Endogenous infections are caused by organism present as part of the normal flora of the patient, while exogenous infections are acquired through exposure to the hospital environment, hospital personnel or medical devices (22). Nosocomial infection rates vary substantially by body site, by type of hospital and by the infection control capabilities of the institution (17). The proportion of infections at each site is also considerably different in each of the major hospital services and by level of patient risk. This is exemplified by surgical site infections (SSIs) which are most common in general survey, whereas urinary tract infections and blood stream infections are most frequent in medical services and nurseries. Rates of nosocomial infection vary by surgical subspecialty, low in ophthalmology and high in general surgery. The differences are largely due to variations in exposure to high risk devices or procedures $(7,31)$.

Urinary tract infections (UTI) represent the most common (34\%) type of nosocomial infections. Indwelling catheters cause the majority while others are caused by genito urinary procedures ${ }^{31}$. Surgical wound infections represent $17 \%$ nosocomial infection and are the second most common hospital acquired infections. The classification of wound infections is based on the degree of bacterial contamination, including clean, clean contaminated and contaminated. Comorbid and contamination of the surgical site contribute to the infection rate. The risk factors for surgical wound infections include age, obesity, concurrent infection and prolonged hospitalizations. The origin of the bacterial agent is dependent on direct inoculation from a host's flora, cross-contamination, the surgeon's hands, air-borne contamination and devices such as drains and catheters ${ }^{31}$. Lower respiratory infection (LRI) or pneumonia represents $13 \%$ of nosocomial infections ${ }^{5}$. This is the most dangerous of all nosocomial infections with a 
case fatality rate of $30 \%$. It manifests in the intensive care unit or post-surgical recovery room. Endotracheal intubation and tracheostomy dry the lower respiratory tract mucous and provide entry for microbes. Other agents that cause nosocomial LRI are ventilators and nebulizers (31). Nosocomial blood stream infections (BSIs) represent $14 \%$ of nosocomial infections (5). Nosocomial bacteremia can be classified as primary or secondary. Primary nosocomial bacteremia occurs without any infection in other sites. Secondary bacteremia is the presence of infection in a site such as urinary tract, surgical wound or lower respiratory tract which can lead to a blood stream infection with the same organism. Mortality from nosocomial bacteremia is greater than primary bacteremia. It is greater than if it is community - acquired. Primary bacteremia or fungaemia usually occurs due to intravenous catheters, intravenous fluid contamination and multidose parenteral medication lines (31). Most blood stream infections are associated with vascular catheter related infections. These catheters may be infected due to contaminated antiseptics used to clean the skin. Contaminated hands of health care personnel, infections following hematogenous seeding or external colonization ${ }^{1}$. The risk factors for peripheral intravenous catheters include duration longer than 72 hours, cut down placement, lower extremity site, urgent placement and poor hand washing. Bacteria pathogens causing community acquired infections differ to some extent from those causing nosocomial infections $(1,31)$.

\section{ETIOLOGY}

Although viruses, fungi and parasites are recognized as souces of nosocomial infections, bacterial agents remain the most commonly recognized cause (32). The offending nosocomial pathogen can depend on the site of the infection. Nosocomial blood stream infections are usually caused by gram-positive organisms including coagulase negative Staphylococcus, Staphylococcus auerus and Enterococci (1). Escherichia coli is a very common cause of nosocomial urinary tract infection, but other pathogens including Pseudomonas aeruginosa, Klebsiella spp, Proteus mirabilis, Staphylococcus epidermidis, Enterococci and Candida spp. Legionella pneumophila may also be responsible for epidemic lower respiratory tract infection in hospitals. Klebsiella spp, Pseudomonas spp, Proteus spp, Escherichia coli and
Staphylococcus aureus are common cause of blood stream nosocomial infections in neonates. 31

The widespread use of broad spectrum antibiotics has led to nosocomial infections with drug resistant microbes (1). Examples include Methicillin resistant Staphylococcus aureus (MRSA) Penicillin resistant precumococci, Vancomycin resistant enterococci, (VRE) and multi drug resistant tuberculosis (MDR-TB). The risk factors for MRSA colonization are overcrowding of wards with limited nursing staff. VRE blood stream infections have been associated with severe illnesses like haematologic malignancies, AIDS, neutropenia, prolonged hospitalization and vancomycin use. Prior use of broad-spectrum antibiotic appears to be a consistent predisposition for drug resistant bacteria. Thus the control and usage of broad spectrum antibiotic is of major importance. MDR-TB has taken place among prison inmates and health care workers $(1,33)$. Hepatitis A, B and $\mathrm{C}$ have been known to be transmitted from patient to health workers and vice versa. The risk of acquiring HIV from a needle stick injuries is $1 / 250(1,33,34)$.

Increasingly aggressive medical and surgical interventions, including implanted foreign bodies, organ transplantations and xenotransplantation, create a cohort of particularly susceptible persons. Renovation of aging hospitals increases risk of air borne fungal and other infections (33).

\section{TRANSMISSION}

Microorganisms are transmitted in hospitals by several routes and same microorganisms may be transmitted by more than one route. The five main routes of transmission includes contact, droplet, airborne, common vehicle and vector borne (1).

Contact transmission is the most important and frequent mode of transmission of nosocomial infections. Direct contact transmission involves a direct body surface-to-body surface contact and physical transfer of micro-organism between a susceptible host and an infected or colonized person such as occurs when a health care worker turns a patient in bed, or gives a bath or performs other patient-care activities that require personal contact. There can also be cross-infection between two patients with one serving as the source of infection and the other as the 
susceptible host. Indirect contact transmission involves contact of a susceptible host with a contaminated intermediate object, usually an instrument such as needle, dressings, or contaminated gloves that are not changed between patients $(1,33)$. Additionally the improper use of saline flush syringes, vials and bags have been implicated in disease transmission in the U.S, even when health care workers had access to gloves, disposable needles, intravenous devices and flushes (1).

Droplet transmission occurs when droplets are generated from the source person mainly during coughing, sneezing and talking and during procedures such as suctioning and bronchoscopy. Transmission occurs when droplets containing microorganisms from an infected person are propelled a short distance through the air and deposited on the host's body. Airborne transmission occurs by dissemination of either airborne droplet nuclei (small particle residue $5 \mu \mathrm{m}$ or smaller in size of evaporated droplet containing microorganisms that remain suspended in the air for long periods of time) or dust particles containing infectious agent. Microorganisms carried in this manner can be dispersed widely by air current and may become inhaled by a susceptible host within the same room or over a long distance from the source patient depending on environmental factors. Examples include Mycobacterium tuberculosis, Legionella, the rubeola and varicella viruses. Common vehicle transmission applies to microorganisms transmitted to the host by contaminated items such as food, water, medications, devices and equipments. Vector borne transmission occurs when vectors such as mosquitoes, flies, rats and other vermin transmit microorganisms $(1,31,33)$.

\section{PREDISPOSITION TO INFECTION}

Within hours of hospital admission, colonies of hospital strains of bacteria develop in patients' skin, respiratory and genitourinary tracts. ${ }^{35}$ Risk factors for the invasion and colonization by pathogens can be categorized into three areas: iatrogenic, organizational, and patient-related factors. Iatrogenic risk factors include invasive procedures (e.g. intubation, indwelling vascular lines, urethral catheterization, blood transfusions) and antibiotic use both active and prophylaxis (35).

Organizational risk factors include contaminated air-conditioning system, contaminated water systems, hospital staff and physical layout of the facility (e.g. nurse-to-patient ratio, open beds close together) (35). Patients' risk factors include the severity of illness, the underlying immunocompromised state and length of stay. Patients already colonized on admission are instantly put at greater risk when they undergo invasive procedures $(35,36)$.

\section{PREVENTION AND CONTROL}

Control measures seek to protect potential sites of infection, interrupt routes of transmission, boost host defenses and discourage selection of hospital strains of organisms ${ }^{22}$. In the hospital, the first step in setting up a viable infection control programme is to set up an infection control committee, which is an essential administrative requirement for effective control of nosocomial infections. The infection control committee is made up of senior administrative staff, i.e. the Chief Medical Director, the infection control doctor, who is often a clinical microbiologist, an epidemiologist or a physician/surgeon with interest in infectious diseases whose opinion is respected, an infection control nurse, heads of clinical departments or their representatives (surgery, medicine, paediatrics, obstetrics and gynaecology etc), representative of nursing staff, pharmacy, engineering, central sterile services department (CSSD). Other co-opted members include representative from catering department, operating theatre, medical supplies and purchasing. (7,37).

The infection control committee should give authority to infection control policies, and to ensure implementation. The principles of infection control are based on the use of common sense and safe practices that prevent or reduce the likelihood of infections being transmitted from a source to a susceptible host ${ }^{37}$ and this include hand washing, which remains the single most important effective measure to reduce the risks of transmitting microbes from one person to another or from one site to another on the same patient $(3,22,29,33)$. Others include use of protective clothing, good personnel and hospital hygiene, adequate management of soiled linen, management of inoculation and contaminated incidents, proper specimen handling and transportation, avoiding spillage on specimen container during collection, proper management of waste generated within the hospital, appropriate use of antiseptics, 
disinfectants and sterilization processes, aseptic techniques in the operating theatre, delivery suites and in the wards, rationale use of antibiotics, surveillance of infection within the hospital by daily collection of data by the infection control team, isolation of highly contagious patients and health education through refresher courses for health workers (37)

\section{CHALLENGES TO THE CONROL MEASURES}

A well-structured infection control programme plays a vital role in reducing mortality, morbidity and cost resulting from nosocomial infections in hospitalized patients. However, infection control activities in developing countries are severely constrained by numerous problems. Most hospitals in developing countries, especially Africa, have no effective infection control programmes and there is lack both of awareness of the problem and of personnel trained in infection control practices (7). Among the difficulties faced that have also been identified as mitigating against an effective infection control programme in the hospital are inadequate and ageing infrastructure including poor water supply, erratic supply of electricity, irregular supply of essential materials like gloves, masks, disinfectants etc, lack of an effective antibiotic policies which is due to the fact that often times these policies are written without due consultation with those that will prescribe and without taking into consideration the antibiotic resistance profile of local organisms, unfortunately, this information is unavailable in many developing nations due to poor or absent antibiotic resistance surveillance programmes which is part of the functions of the infection control team, poor funding of nosocomial infection programme, poor laboratory backup, lack of awareness of the activities of the infection control team or the cost benefits of an efficient control programme, the presence of family members in hospital, limited isolation facilities and in some cases patient presenting in advanced state of the disease process rendering isolation difficult $(7,28,29,33,37,38,39)$.

Although effective hand washing programme still remains the single most important procedure in reducing cross infection, in developing countries, like Nigeria, this is usually difficult because there is often no running water with which to wash hands such that many facilities resort to a "bucket" and "bowl" with consequent contamination. Even as simple as it seems to be, it is often not used or performed incorrectly (39) Surveillance is the backbone of a good infection control programme, but studies have shown that nosocomial infection surveillance and control have met little success in developing countries such as Nigeria due to non functional infection control committee or there is no infection control programme (39). In many industrialized countries, apart from the hospital infection control committee, there exist national bodies like Centre for Disease Control(CDC), National Nosocomial Infections Surveillance(NNIS) in the USA ,that collate and analyze data on hospital infection nationwide. No such institution exists in Nigeria though there is an urgent need for it (7). The solutions to these problems will include setting up an infection control committee with the powers to implement infection control policies. In formulating policies, the committee should ensure wide consultations, identify interested ward personnel, study the existing practices, formulate and implement simple, easyto-follow policies, which should be incorporated in written policies in the infection control manual. The cost of hospital infection control programme must be considered an essential part of the health budget of the country and there should be a fund allocation for the infection control committee for routine control purposes and a contingency fund to bear the costs of outbreak $(7,37)$.

\section{COST-EFFECTIVE APPLICATION OF MOLECULAR TYPING}

The use of phenotypic methods for the characterization of nosocomial pathogens has been useful for the understanding of pathogens,; however, these methods have drawbacks that limit their utility for highly discriminatory typing of microorganisms. Limitations of serotyping include lack of availability of certain antisera and problems with standardization of different methods. Biotyping often lacks discriminatory power because of variations in gene expression and random mutations that may alter biologic properties of microorganisms. Bacteriophage typing is labor-intensive and method often demonstrates poor reproducibility and standardization. Bacteriocin typing may have some utility for organisms not easily typed, such as Pseudomonas aeruginosa and Candida species. Molecular typing methods including Plasmid Analysis, Southern Blot AnalysisRibotyping, Pulse-field gel electrophoresis(PFGE), Amplified fragment 
length polymorphism (AFLP) and Polymerase Chain Reaction (PCR) are powerful tools in the armamentarium for combating the spread of problematic microorganisms in the hospital environment (.41). The integration of molecular typing with conventional hospital epidemiologic surveillance has been proven to be cost-effective due to an associated reduction in the number of nosocomial infections . $(41,42,43)$

development of treatment regimens to manage patients affected by related organisms. Therefore, the use of molecular tests is essential in many circumstances for establishing disease epidemiology, which leads to improved patient health and economic benefits through reduction of nosocomial infections (41) Recent information also suggests the use of an integrated laboratory assessment of drugresistant pathogens to have an impact on rates of endemic infection and can be cost saving (43)

\section{CONCLUSION/RECOMMMENDATION}

Nosocomial infections may be contained by having an effective control programme with computer-assisted epidemiological surveillance for monitoring which should be handled as a global project with significant inputs from developing countries.

There is urgent need for review and boost of the prevailing infection control measures in various hospitals particularly in developing countries.

\section{REFERENCES}

1. Nosocomial infection. http:// en.wikipedia. org / wiki / nosoconial_ infection.

Retrieved $9^{\text {th }}$ June, 2009.

2. Horan T.C, Gaynes R.P. Surveillance of nosocomial infections. In: Hospital epidemiology and infection control $3^{\text {rd }}$ ed., Mayhall C.G (ed).Philadelphia Lippincortt. Williams and Wilkins,2004: 1659-1702

3. World Health Organization. Meeting on hospital infection prevalence, Geneva. WHO/MIN/NIC/871. 20-22, Oct, 1986.

4. Ricks, Delthia, "Germ warfare". Ms. Magazine spring 2007. pp 43-45.

5. Taiwo SS, Onile BA, Akanbi II AA. Methicillin-Resistant Staphylococcus aureus(MRSA)isolates in Ilorin, Nigeria. Afr.J.Clin.Exper.Microbiol. 2004;5(2):189-197.

6. Robert AW. Controlling Antimicrobial resistance in hospitals infection control
Molecular techniques can be very effective in tracking the spread of nosocomial infections due to genetically related pathogens, which would allow infection control personnel to more rationally identify potential sources of pathogens and aid infectious disease physicians in the

The continuous education of hospital authorities and health workers on principles of infection control through training and re-training is advocated. Infection control should be incorporated into the curriculum of medical/dental students, student nurses and other paramedical. There is need for adequate employment of health workers especially nurses on hospital wards since overworked staff may become ineffective and this can result in epidemic infections in some situations. Major advances in overall control of infectious diseases have resulted from immunization, improved hygiene, particularly hand washing. Health care settings must continually remind hospital personnel on better implementation of existing infection control programmes especially hand washing so that we will not need to rely solely on technological advances in order to bring nosocomial infections to the barest minimal level.

and use of antibiotics. Emerg Infect Dis. 2001; 7(2): 188-192.

7. Tolu Odugbemi. Setting up An infection control programmes in the Hospital: Role of the policy-makers.

Journal of the Nigeria Infection Association. 1999; 2(1): 4-8

8. Semmelweis IP. Vortroy uber die genesis des puerperal fiebers, vom 15 May 1850, in milestones in Microbiology Brock, T.D ed. Asm, Washington D.C 80.

9. Thomas CGA. Control of hospital and community infections. In Medical Microbiology $6^{\text {th }}$ ed. Bailliere Tindall, W.B. saunders, 1988, P. 64-68.

10. Lister J. on a new method of treating compound fractures, abscess and soforth, with observations on the conditions of suppuration. Lancet 1867 , $1: 326,357,387,507$ and 295. 
11. Mehtar S. importance of infection control in wenzel R, Edmond M, Pittet D, Devaster JM, Brewer T, Geddes A, Butzer JP. (ed). A guide to infection control in the Hospital. B.C. Decker Inc. Hamilton London, 1998; P. 1-4

12. Proceedings of the National conferences on Hospital-acquired staphylococcal disease, US Department of Health, Education and welfare communicable Disease canter Atlantic, Ga, 1958.

13. Joint commission on Accreditation of Hospitals. Bulletin of the joint commission on Accreditation of Hospitals, N0 18, 1958.

14. Gardner AMN, Stamp M, Bowgen JA, Moore B. the infection control sister. A new member of the control of infection team in general hospitals. Lancet 1962; 11:710.

15. Moore B. the infection control sister in British hospitals. Int nurs Rev 1970; $17: 84$

16. Wenzel R. the infection control nurse Nurs clin North Am 1970; 89

17. Italey Riv, Schachtman RH. The emergence of infection surveillance and control programmes in US. Hospitals: an assessment, 1976.

18. Centers for Disease control. Nosocomial infection surveillance, 1984. in surveillance summarizes 1986; 35 (N0. 155): 17-29.

19. Garner JS, Jarvis we, Emori TG, Horan TC, Hughes JM. CDC definitions. I for nosocomial infections, 1988. Am $1.1 \mathrm{~J}$ infect control. 1988 16; 128-140.

20. Hughes Jm, Jarvis WR. Epidemiology of nosocomial infections. In Lennets EH, Balows A, Hausler wj Jr. Shadomy HJ (ed) manual of clinical microbiology $4^{\text {th }}$ ed. Asm, Washington D.C 1985; p99-104.

21. Pechere JC. Microbiology of nosocomial infections. Bull Acad Natl Med. 1993; 177 (5): 705-715.

22. Leroy Edozein. Denying microorganisms their chance Africa Health May 1992; 14 (4): 37-38.

23. Alausa KO, Onile BA. Epidemiological pattern of bacterial septicaemia at the university College Hospital Ibadan. Nig. Med. Jour. 1984; 14 (1\&2): 55-62.

24. Klevens RM, Edwards JR, Richards CL. Estimating health care-associated infections and deaths in U.S. hospitals. Public Health Rep. 2007;122 (2) :160166.

25. Supplemental lecture (98/05/09 update) by Stephen T. Abedon. http: // www. Mansfield. Ohio- state. Edu/ sabed on /bio/2053. htm. Retieved 6/10/2007.

26. L'Italie scandalisee par ccl' hospital d I'horreur" Eric Jozsef, Liberation. 15 Janvier 2007.

27.

http/www.swishandhygiene.ch /swisshandhygiene/presse/b/content files/301006_facts_sheet_F doc. Retrieved 6/10/2007

28. Onipede AO,Oluyede CO,Aboderin $\mathrm{AO}$, et al.A survey of hospital acquired infections in Obafemi Awolowo University Teaching Hospital, Ile-Ife. Afri .J. Clin. Experi. Microbiol. 2004; 5(1): 108-118.

29. Kesah CN, Egri-Okwaji MTC, Odugbemi TO, Iroha EO. Bacteria associated with

nosocomial infection and their antimicrobial pattern in pediatric patients in a

tertiary health institution. J Med. Med Sci.1999;1:6-13.

30. Odimayo M.S, Nwabuisi C., Adegboro B. Hospital acquired Infections in Nigeria. Tropical Journal of Health Sciences. 2008;

15(1) : 49-54.

31. Nosocomial infections and infection control inhospital. http: //complab.nymc.edu/Curriculum/ComPr evMed/Nosocomial Infections.htm. Retrieved on 6/10/2007.

32. Dembri L, Zervos MJ, Heirholzer WJ. 1998. Nosocomial bacterial infections, $3^{\text {rd }}$ ed.,p.501-528. In E.A Brachman (ed.). Bacterial infection in humans. Plenum Publishing Corporation, New York. N.Y.

33. Weistein RA. Nosocomial infection update. Emerg. Infect. Dis. 1998. 4(3):416-420

34. Medubi SA, Akande TM, Osagbemi GK. Awareness and pattern of needle stick injuries among health workers at university of Ilorin teaching Hospital, Ilorin, Nigeria. Afr. J. clin. Exp. Microbiol. 2006. 7(3): 183-87 
35. C: /My by Quoc V Nguyen, MD..htm22/12/2004.

36. Julie Louise Gerberding. HospitalOnset infections: A patient safety issue. An. Int Med. 2002;137: 665-670.

37. Shaheen Mehtar. Hospital infection control:Setting up a cost-effective programme, $1^{\text {st }}$ ed. Oxford University Press, New York; 1992 : 17-25.

38. Scott GM. Infection control with limited resources. Africa Health. 1990; 13 (1):40-43.

39. Ogunsola FT,Oduyebo O,Iregbu $\mathrm{KC}$,Coker AO,Adetunji A. Areview of nosocomial infections at Lagos University Teaching Hospital:Problems and strategies for improvement. Journal of the Nigerian infection Association.1998;1(1):14-20.
40. Nyamogoba H., Obala AA. Nosocomial infections in developing countries: Cost effective control and prevention. East Afr Med J.2002; 79(8) : 435-441.

41. Singh A, Goering RV, Simjee S, Foley SL, Zervos MJ. Application of molecular techniques to the study of hospital infection. Clin Microbiol Rev .2006; 19(3) :512-530.

42. Diekema DJ, Dogson KL, Sigurdardottir B, Pfaller MA. Rapid detection of antimicrobial-resistant organism carriage: an unmet clinical need. J. Clin. Microbiol.2004;42 : 28792883.

43. Peterson LR, Brossette SE. Hunting health care-associated infections from the clinical microbiology laboratory: passive, active and virtual surveillance. J. Clin. Microbiol. 2002;40 : 1-4. 